\title{
Continuity for multilinear commutator of Marcinkiewicz operator on Besov spaces
}

\author{
Gu Guangze and Cai Mingjie
}




\title{
CONTINUITY FOR MULTILINEAR COMMUTATOR OF MARCINKIEWICZ OPERATOR ON BESOV SPACES
}

\author{
GU GUANGZE AND CAI MINGJIE \\ Received 24 September, 2013
}

\begin{abstract}
In this paper we prove the continuity for the multilinear commutator associated to the Marcinkiewicz operator on Besov spaces.

2010 Mathematics Subject Classification: 42B20; 42B25

Keywords: multilinear commutator, Marcinkiewicz operator, Besov space
\end{abstract}

\section{INTRODUCTION}

As the development of singular integral operators, their commutators and multilinear operators have been well studied (see [1-4, 14]). From $[2,4,9,13]$, we know that the commutators and multilinear operators generated by singular integral operators and the Lipschitz functions are bounded on the Triebel-Lizorkin and Lebesgue spaces. The purpose of this paper is to introduce the multilinear commutator associated to the Marcinkiewicz operator and prove the continuity properties for the multilinear commutator on Besov spaces.

\section{PRELIMINARIES AND THEOREMS}

First, let us introduce some notations. Throughout this paper, $Q$ will denote a cube of $R^{n}$ with sides parallel to the axes. For a locally integrable function $f$, the sharp function of $f$ is defined by

$$
f^{\#}(x)=\sup _{Q \ni x} \frac{1}{|Q|} \int_{Q}\left|f(y)-f_{Q}\right| d y,
$$

where, and in what follows, $f_{Q}=|Q|^{-1} \int_{Q} f(x) d x$. It is well-known that (see $[14,15])$

$$
f^{\#}(x) \approx \sup _{Q \ni x} \inf _{c \in C} \frac{1}{|Q|} \int_{Q}|f(y)-c| d y .
$$

(c) 2015 Miskolc University Press 
For $\beta \geq 0$, the Besov space $\dot{\lambda}_{\beta}\left(R^{n}\right)$ is the space of functions $f$ such that

$$
\|f\|_{\dot{\lambda}_{\beta}}=\sup _{\substack{x, h \in R^{n} \\ h \neq 0}}\left|\Delta_{h}^{[\beta]+1} f(x)\right| /|h|^{\beta}<\infty,
$$

where $\Delta_{h}^{k}$ denotes the $k$-th difference operator (see [12]).

For $b_{j} \in \dot{\wedge}_{\beta}\left(R^{n}\right)(j=1, \cdots, m)$, set

$$
\|\vec{b}\|_{\dot{\wedge}_{\beta}}=\prod_{j=1}^{m}\left\|b_{j}\right\|_{\dot{\wedge}_{\beta}} .
$$

Given some functions $b_{j}(j=1, \cdots, m)$ and a positive integer $m$ and $1 \leq j \leq m$, we denote by $C_{j}^{m}$ the family of all finite subsets $\sigma=\{\sigma(1), \cdots, \sigma(j)\}$ of $\{1, \cdots, m\}$ of $j$ different elements. For $\sigma \in C_{j}^{m}$, set $\sigma^{c}=\{1, \cdots, m\} \backslash \sigma$. For $\vec{b}=\left(b_{1}, \cdots, b_{m}\right)$ and $\sigma=\{\sigma(1), \cdots, \sigma(j)\} \in C_{j}^{m}$, set $\vec{b}_{\sigma}=\left(b_{\sigma(1)}, \cdots, b_{\sigma(j)}\right), b_{\sigma}=b_{\sigma(1)} \cdots b_{\sigma(j)}$ and $\left\|\vec{b}_{\sigma}\right\|_{\dot{\lambda}_{\beta}}=\left\|b_{\sigma(1)}\right\|_{\dot{\lambda}_{\beta}} \cdots\left\|b_{\sigma(j)}\right\|_{\dot{\lambda}_{\beta}}$.

Definition 1. Let $0<p, q \leq \infty, \alpha \in R$. For $k \in Z$, set $B_{k}=\left\{x \in R^{n}:|x| \leq 2^{k}\right\}$ and $C_{k}=B_{k} \backslash B_{k-1}$. Denote by $\chi_{k}$ the characteristic function of $C_{k}$ and $\chi_{0}$ the characteristic function of $B_{0}$.

(1) The homogeneous Herz space is defined by

$$
\dot{K}_{q}^{\alpha, p}\left(R^{n}\right)=\left\{f \in L_{l o c}^{q}\left(R^{n} \backslash\{0\}\right):\|f\|_{\left.\dot{K}_{q}^{\alpha, p}<\infty\right\},}\right.
$$

where

$$
\|f\|_{\dot{K}_{q}^{\alpha, p}}=\left[\sum_{k=-\infty}^{\infty} 2^{k \alpha p}\left\|f \chi_{k}\right\|_{L^{q}}^{p}\right]^{1 / p}
$$

(2) The nonhomogeneous Herz space is defined by

$$
K_{q}^{\alpha, p}\left(R^{n}\right)=\left\{f \in L_{l o c}^{q}\left(R^{n}\right):\|f\|_{\left.K_{q}^{\alpha, p}<\infty\right\},}\right.
$$

where

$$
\|f\|_{K_{q}^{\alpha, p}}=\left[\sum_{k=1}^{\infty} 2^{k \alpha p}\left\|f \chi_{k}\right\|_{L^{q}}^{p}+\left\|f \chi_{B_{0}}\right\|_{L^{q}}^{p}\right]^{1 / p}
$$

And the usual modification is made when $p=q=\infty$.

Definition 2. Let $1 \leq q<\infty, \alpha \in R$. The central Campanato space is defined by (see [17])

$$
C L_{\alpha, q}\left(R^{n}\right)=\left\{f \in L_{l o c}^{q}\left(R^{n}\right):\|f\|_{C L_{\alpha, q}}<\infty\right\},
$$

where

$$
\|f\|_{C L_{\alpha, q}}=\sup _{r>0}|B(0, r)|^{-\alpha}\left(\frac{1}{|B(0, r)|} \int_{B(0, r)}\left|f(x)-f_{B(0, r)}\right|^{q} d x\right)^{1 / q} .
$$


Definition 3. Let $0<\delta<n, 0<\gamma \leq 1$ and $\Omega$ be homogeneous of degree zero on $R^{n}$ such that $\int_{S^{n-1}} \Omega\left(x^{\prime}\right) d \sigma\left(x^{\prime}\right)=0$. Assume that $\Omega \in L i p_{\gamma}\left(S^{n-1}\right)$, that is there exists a constant $M>0$ such that for any $x, y \in S^{n-1},|\Omega(x)-\Omega(y)| \leq M|x-y|^{\gamma}$. The Marcinkiewicz multilinear commutator is defined by

$$
\mu_{\Omega, \delta}^{\vec{b}}(f)(x)=\left(\int_{0}^{\infty}\left|F_{t, \delta}^{\vec{b}}(f)(x)\right|^{2} \frac{d t}{t^{3}}\right)^{1 / 2},
$$

where

$$
F_{t, \delta}^{\vec{b}}(f)(x)=\int_{|x-y| \leq t} \frac{\Omega(x-y)}{|x-y|^{n-1-\delta}}\left[\prod_{j=1}^{m}\left(b_{j}(x)-b_{j}(y)\right)\right] f(y) d y .
$$

Set

$$
F_{t, \delta}(f)(x)=\int_{|x-y| \leq t} \frac{\Omega(x-y)}{|x-y|^{n-1-\delta}} f(y) d y,
$$

we also define that

$$
\mu_{\Omega, \delta}(f)(x)=\left(\int_{0}^{\infty}\left|F_{t, \delta}(f)(x)\right|^{2} \frac{d t}{t^{3}}\right)^{1 / 2},
$$

which is the Marcinkiewicz operator (see [16]). that

Let $H$ be the space $H=\left\{h:\|h\|=\left(\int_{0}^{\infty}|h(t)|^{2} \frac{d t}{t^{3}}\right)^{1 / 2}<\infty\right\}$. Then, it is clear

$$
\mu_{\Omega, \delta}(f)(x)=\left\|F_{t, \delta}(f)(x)\right\| \text { and } \mu_{\Omega, \delta}^{\vec{b}}(f)(x)=\left\|F_{t, \delta}^{\vec{b}}(f)(x)\right\| .
$$

Note that when $b_{1}=\cdots=b_{m}, \mu_{\Omega, \delta}^{\vec{b}}$ is just the $m$ order commutator. It is well known that commutators are of great interest in harmonic analysis and have been widely studied by many authors (see $[1-9,11-13]$ ). Our main purpose is to study the boundedness properties for the multilinear commutator $\mu_{\Omega, \delta}^{\vec{b}}$ on Besov spaces.

Now we state our theorems as following.

Theorem 1. Let $0<\delta<n, \quad 1<r<n / \delta, 1 / s=1 / r-\delta / n, \quad 0<\beta$ $<\min (1 / 2 m, \gamma / m)$ for $0<\gamma \leq 1$, and $b_{j} \in \dot{\lambda}_{\beta}\left(R^{n}\right)$ for $j=1, \cdots, m$. Then $\mu_{\Omega, \delta}^{\vec{b}}$ is bounded from $L^{p}\left(R^{n}\right)$ to $\dot{\wedge}_{\delta+m \beta-n / p}\left(R^{n}\right)$ for any $n /(\delta+m \beta) \leq p \leq n / \delta$.

Theorem 2. Let $0<\delta<n, 0<\beta<\min (1 / 2 m, \gamma / m)$ for $0<\gamma \leq 1,1<q_{1}<$ $n /(\delta+m \beta), 1 / q_{2}=1 / q_{1}-(\delta+m \beta) / n, \max \left(-n / q_{2}-1 / 2,-n / q_{2}-\gamma\right)<\alpha \leq$ $-n / q_{2}$ and $b_{j} \in \dot{\wedge}_{\beta}\left(R^{n}\right)$ for $j=1, \cdots, m$. Then $\mu_{\Omega, \delta}^{\vec{b}}$ is bounded from $\dot{K}_{q_{1}}^{\alpha, \infty}\left(R^{n}\right)$ to $C L_{-\alpha / n-1 / q_{2}, q_{2}}\left(R^{n}\right)$.

Remark 1. Theorem 2 also hold for the nonhomogeneous Herz type Hardy space. 


\section{Proofs of THEOREMS}

To prove the theorems, we need the following lemmas.

Lemma 1 ([12]). For $0<\beta<1,1 \leq p \leq \infty$, we have

$$
\begin{aligned}
\|b\|_{\dot{\lambda}_{\beta}} & \approx \sup _{Q} \frac{1}{|Q|^{1+\beta / n}} \int_{Q}\left|b(x)-b_{Q}\right| d x \approx \sup _{Q} \frac{1}{|Q|^{\beta / n}}\left(\frac{1}{|Q|} \int_{Q}\left|b(x)-b_{Q}\right|^{p} d x\right)^{1 / p} \\
& \approx \operatorname{supinf}_{Q} \frac{1}{|Q|^{1+\beta / n}} \int_{Q}|b(x)-c| d x \approx \operatorname{supinf}_{Q} \frac{1}{|Q|^{\beta / n}}\left(\frac{1}{|Q|} \int_{Q}|b(x)-c|^{p} d x\right)^{1 / p} .
\end{aligned}
$$

Lemma 2 ([10]). For $\alpha<0,0<q<\infty$, we have

$$
\|f\|_{\dot{K}_{q}^{\alpha, \infty}} \approx \sup _{\mu \in Z} 2^{\mu \alpha}\left\|f \chi_{B_{\mu}}\right\|_{L^{q}} .
$$

Lemma 3 ([10]). Let $0<\eta<n, 1<p<n / \eta$. Suppose $b \in \dot{\wedge}_{\beta}\left(R^{n}\right)$, then

$$
\left|b_{2^{k+1} B}-b_{B}\right| \leq C|| b \|_{\dot{\lambda}_{\beta}} k\left|2^{k+1} B\right|^{\beta / n} \text { for } k \geq 1 .
$$

Lemma 4 ([16]). Let $0<\delta<n, 1<p<n / \delta$ and $1 / q=1 / p-\delta / n$. Then $\mu_{\Omega, \delta}$ is bounded from $L^{p}\left(R^{n}\right)$ to $L^{q}\left(R^{n}\right)$.

Lemma 5 ([7]). Let $0 \leq \eta<n, 1<r<n / \eta, 1 / r-1 / s=\eta / n$ and $b_{j} \in \dot{\wedge}_{\beta}\left(R^{n}\right)$ for $j=1, \cdots, m$. Then $\mu_{\Omega, \delta}^{\vec{b}}$ is bounded from $L^{r}\left(R^{n}\right)$ to $L^{s}\left(R^{n}\right)$.

Proof of Theorem 1. It is only to prove that there exists a constant $C_{0}$ such that

$$
\frac{1}{|Q|^{1+(\delta+m \beta) / n-1 / p}} \int_{Q}\left|\mu_{\Omega, \delta}^{\vec{b}}(f)(x)-C_{0}\right| d x \leq C\|f\|_{L^{p}}
$$

Fix a cube $Q, Q=Q\left(x_{0}, d\right)$, we decompose $f$ into $f=f_{1}+f_{2}$ with $f_{1}=f \chi_{Q}$, $f_{2}=f \chi\left(R^{n} \backslash Q\right)$.

When $m=1$, for $C_{0}=\mu_{\Omega, \delta}\left(\left(\left(b_{1}\right)_{Q}-b_{1}\right) f_{2}\right)\left(x_{0}\right)$, we have

$$
\begin{aligned}
F_{t, \delta}^{b_{1}}(f)(x)= & \left(b_{1}(x)-\left(b_{1}\right)_{Q}\right) F_{t, \delta}(f)(x) \\
& -F_{t, \delta}\left(\left(b_{1}-\left(b_{1}\right)_{Q}\right) f_{1}\right)(x)-F_{t, \delta}\left(\left(b_{1}-\left(b_{1}\right)_{Q}\right) f_{2}\right)(x) .
\end{aligned}
$$

Then

$$
\begin{aligned}
\mid & \mu_{\Omega, \delta}^{b_{1}}(f)(x)-\mu_{\Omega, \delta}\left(\left(\left(b_{1}\right)_{Q}-b_{1}\right) f_{2}\right)\left(x_{0}\right) \mid \\
= & \left|\left\|F_{t, \delta}^{b_{1}}(f)(x)\right\|-\left\|F_{t, \delta}\left(\left(\left(b_{1}\right)_{Q}-b_{1}\right) f_{2}\right)\left(x_{0}\right)\right\|\right| \\
\leq & \left\|\left(b_{1}(x)-\left(b_{1}\right)_{Q}\right) F_{t, \delta}(f)(x)\right\|+\left\|F_{t, \delta}\left(\left(b_{1}-\left(b_{1}\right) Q\right) f_{1}\right)(x)\right\| \\
& +\left\|F_{t, \delta}\left(\left(b_{1}-\left(b_{1}\right) Q\right) f_{2}\right)(x)-F_{t, \delta}\left(\left(b_{1}-\left(b_{1}\right) Q\right) f_{2}\right)\left(x_{0}\right)\right\| \\
= & A(x)+B(x)+C(x) .
\end{aligned}
$$


For $A(x)$, for $1<p<q<n / \delta, 1 / q=1 / p-\delta / n$, by the boundness of $\mu_{\Omega, \delta}$ from $L^{p}\left(R^{n}\right)$ to $L^{q}\left(R^{n}\right)$ and Hölder's inequality with exponent $1 / q+1 / q^{\prime}=1$ and Lemma 1 , we have

$$
\begin{aligned}
& \frac{1}{|Q|^{1+(\delta+\beta) / n-1 / p}} \int_{Q}|A(x)| d x \\
& \leq \frac{1}{|Q|^{1+(\delta+\beta) / n-1 / p}}\left(\int_{Q}\left|b_{1}(x)-\left(b_{1}\right)_{Q}\right|^{q^{\prime}} d x\right)^{1 / q^{\prime}}\left(\int_{Q}\left|\mu_{\Omega, \delta}(f)(x)\right|^{q} d x\right)^{1 / q} \\
& \leq C \frac{|Q|^{\beta / n+1 / q^{\prime}}}{|Q|^{1+(\delta+\beta) / n-1 / p}} \frac{1}{|Q|^{\beta / n}}\left(\frac{1}{|Q|} \int_{Q}\left|b_{1}(x)-\left(b_{1}\right)_{Q}\right|^{q^{\prime}} d x\right)^{1 / q^{\prime}}\left(\int_{Q}|f(x)|^{p} d x\right)^{1 / p} \\
& \leq C|| b_{1}||_{\dot{\lambda}_{\beta}}|| f \|_{L^{p}} .
\end{aligned}
$$

For $B(x)$, denoting $p=r t, 1<r<s<n / \delta, 1 / s=1 / r-\delta / n$, by the boundness of $\mu_{\Omega, \delta}$ from $L^{r}\left(R^{n}\right)$ to $L^{s}\left(R^{n}\right)$ and Hölder's inequality with exponent $1 / t+1 / t^{\prime}=1$ and Lemma 1 , we have

$$
\begin{aligned}
& \frac{1}{|Q|^{1+(\delta+\beta) / n-1 / p}} \int_{Q}|B(x)| d x \\
& \leq \frac{1}{|Q|^{(\delta+\beta) / n-1 / p}}\left(\frac{1}{|Q|} \int_{R^{n}}\left|\mu_{\Omega, \delta}\left(\left(b_{1}(x)-\left(b_{1}\right)_{Q}\right) f \chi_{Q}\right)(x)\right|^{s} d x\right)^{1 / s} \\
& \leq C \frac{1}{|Q|^{(\delta+\beta) / n-1 / p+1 / s}}\left(\int_{Q}\left|\left(b_{1}(x)-\left(b_{1}\right)_{Q}\right) f(x)\right|^{r} d x\right)^{1 / r} \\
& \leq C \frac{1}{|Q|^{(\delta+\beta) / n-1 / p+1 / s}}\left(\int_{Q}\left|b_{1}(x)-\left(b_{1}\right)_{Q}\right|^{r t^{\prime}} d x\right)^{1 / r t^{\prime}}\left(\int_{Q}|f(x)|^{r t} d x\right)^{1 / r t} \\
& \leq\left. C|| b_{1}\right|_{\lambda_{\beta}}|| f \|_{L^{p}} .
\end{aligned}
$$

For $C(x)$, note that $\left|x_{0}-y\right| \approx|x-y|$ for $y \in Q^{c}$, we have

$$
\begin{aligned}
C(x)= & \| F_{t, \delta}\left(\left(b_{1}-\left(b_{1}\right)_{Q}\right) f_{2}\right)(x)-F_{t, \delta}\left(\left(b_{1}-\left(b_{1}\right)_{Q}\right) f_{2}\right)\left(x_{0}\right)|| \\
= & \left(\int_{0}^{\infty} \mid \int_{|x-y| \leq t} \frac{\Omega(x-y) f_{2}(y)}{|x-y|^{n-1-\delta}}\left(b_{1}(y)-\left(b_{1}\right)_{Q}\right) d y\right. \\
& \left.-\left.\int_{\left|x_{0}-y\right| \leq t} \frac{\Omega\left(x_{0}-y\right) f_{2}(y)}{\left|x_{0}-y\right|^{n-1-\delta}}\left(b_{1}(y)-\left(b_{1}\right)_{Q}\right) d y\right|^{2} \frac{d t}{t^{3}}\right)^{1 / 2} \\
\leq & \left(\int_{0}^{\infty}\left[\int_{|x-y| \leq t,\left|x_{0}-y\right|>t} \frac{|\Omega(x-y)|\left|f_{2}(y)\right|}{|x-y|^{n-1-\delta}}\left|\left(b_{1}(y)-\left(b_{1}\right)_{Q}\right)\right| d y\right]^{2} \frac{d t}{t^{3}}\right)^{1 / 2} \\
& +\left(\int_{0}^{\infty}\left[\int_{|x-y|>t,\left|x_{0}-y\right| \leq t} \frac{\left|\Omega\left(x_{0}-y\right)\right|\left|f_{2}(y)\right|}{\left|x_{0}-y\right|^{n-1-\delta}}\left|b_{1}(y)-\left(b_{1}\right)_{Q}\right| d y\right]^{2} \frac{d t}{t^{3}}\right)^{1 / 2} \\
& +\left(\int _ { 0 } ^ { \infty } \left[\int_{|x-y| \leq t,\left|x_{0}-y\right| \leq t} \frac{|\Omega(x-y)|}{|x-y|^{n-1-\delta}}-\frac{\left|\Omega\left(x_{0}-y\right)\right|}{\left|x_{0}-y\right|^{n-1-\delta} \mid} \mid\right.\right.
\end{aligned}
$$




$$
\begin{aligned}
& \left.\left.\times\left|b_{1}(y)-\left(b_{1}\right)_{Q}\right|\left|f_{2}(y)\right| d y\right]^{2} \frac{d t}{t^{3}}\right)^{1 / 2} \\
\equiv & I_{1}+I_{2}+I_{3} .
\end{aligned}
$$

By the Minkowski's inequality and Hölder's inequality and Lemmas 1, 3:

$$
\begin{aligned}
& I_{1} \leq C \int_{Q^{c}}\left|b_{1}(y)-\left(b_{1}\right)_{Q}\right| \frac{|f(y)|}{|x-y|^{n-1-\delta}}\left(\int_{|x-y| \leq t<\left|x_{0}-y\right|} \frac{d t}{t^{3}}\right)^{1 / 2} d y \\
& \leq C \int_{Q^{c}}\left|b_{1}(y)-\left(b_{1}\right)_{Q}\right| \frac{|f(y)|}{|x-y|^{n-1-\delta}}\left|\frac{1}{|x-y|^{2}}-\frac{1}{\left|x_{0}-y\right|^{2}}\right|^{1 / 2} d y \\
& \leq C \sum_{k=0}^{\infty} \int_{2^{k+1} Q \backslash 2^{k} Q}\left|b_{1}(y)-\left(b_{1}\right)_{2 Q}\right|\left|x_{0}-y\right|^{\delta} \frac{|Q|^{1 / 2 n}|f(y)|}{\left|x_{0}-y\right|^{n+1 / 2}} d y \\
& \leq C \sum_{k=0}^{\infty} 2^{-k / 2} \frac{1}{\left|2^{k+1} Q\right|^{1-\delta / n}}\left(\int_{2^{k+1} Q}|f(y)|^{p} d y\right)^{1 / p}
\end{aligned}
$$

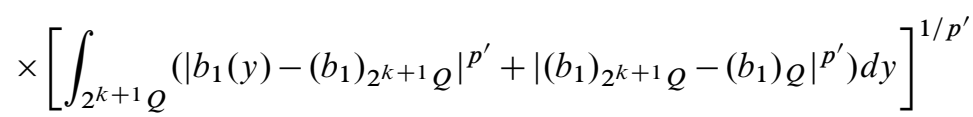

$$
\begin{aligned}
& \leq C \sum_{k=0}^{\infty} 2^{-k / 2} \frac{1}{\left|2^{k+1} Q\right|^{1-\delta / n}}\left(\int_{2^{k+1} Q}|f(y)|^{p} d y\right)^{1 / p} \\
& \times\left[\left|2^{k+1} Q\right|^{\beta / n+1 / p^{\prime}} \frac{1}{\left|2^{k+1} Q\right|^{\beta / n}}\left(\frac{1}{\left|2^{k+1} Q\right|} \int_{2^{k+1} Q}\left|b_{1}(y)-\left(b_{1}\right)_{2^{k+1}} Q\right|^{p^{\prime}} d y\right)^{1 / p^{\prime}}\right. \\
& \left.+\left|\left(b_{1}\right)_{2^{k+1}} Q-\left(b_{1}\right)_{Q}\right|\left|2^{k+1} Q\right|^{1 / p^{\prime}}\right] \\
& \leq\left. C \sum_{k=0}^{\infty} k 2^{k(-1 / 2+\delta+\beta-n / p)}|Q|^{\beta / n+1 / p^{\prime}-1+\delta / n}\left\|b_{1}\right\|\right|_{\lambda_{\beta}}\|f\|_{L^{p}} \\
& \leq C|Q|^{(\delta+\beta) / n-1 / p} \mid\left\|b_{1}\right\|_{\dot{\lambda}_{\beta}}\|f\|_{L^{p}} .
\end{aligned}
$$

Similarly, we have $I_{2} \leq C|Q|^{(\delta+\beta) / n-1 / p}\left\||| b_{1}\right\|_{\dot{\lambda}_{\beta}}|| f \|_{L^{p}}$.

We now estimate $I_{3}$. By the following inequality:

$$
\left|\frac{\Omega(x-y)}{|x-y|^{n-1-\delta}}-\frac{\Omega\left(x_{0}-y\right)}{\left|x_{0}-y\right|^{n-1-\delta}}\right| \leq C\left(\frac{\left|x-x_{0}\right|}{\left|x_{0}-y\right|^{n-\delta}}+\frac{\left|x-x_{0}\right|^{\gamma}}{\left|x_{0}-y\right|^{n-1-\delta+\gamma}}\right),
$$

we obtain

$$
\begin{aligned}
I_{3} \leq & C \int_{Q^{c}}\left|b_{1}(y)-\left(b_{1}\right)_{Q}\right| \frac{|f(y)|\left|x-x_{0}\right|}{\left|x_{0}-y\right|^{n-\delta}}\left(\int_{\left|x_{0}-y\right| \leq t,|x-y| \leq t} \frac{d t}{t^{3}}\right)^{1 / 2} d y \\
& +C \int_{Q^{c}}\left|b_{1}(y)-\left(b_{1}\right)_{Q}\right| \frac{|f(y)|\left|x-x_{0}\right|^{\gamma}}{\left|x_{0}-y\right|^{n-1-\delta+\gamma}}\left(\int_{\left|x_{0}-y\right| \leq t,|x-y| \leq t} \frac{d t}{t^{3}}\right)^{1 / 2} d y \\
\leq & C \sum_{k=0}^{\infty} \int_{2^{k+1} Q \backslash 2^{k} Q}\left|b_{1}(y)-\left(b_{1}\right)_{Q}\right|\left(\frac{|Q|^{1 / n}}{\left|x_{0}-y\right|^{n+1-\delta}}+\frac{|Q|^{\gamma / n}}{\left|x_{0}-y\right|^{n+\gamma-\delta}}\right)|f(y)| d y
\end{aligned}
$$




$$
\begin{aligned}
\leq & C \sum_{k=0}^{\infty}\left(2^{-k}+2^{-k \gamma}\right) \frac{1}{\left|2^{k+1} Q\right|^{1-\delta / n}} \int_{2^{k+1} Q}\left|b_{1}(y)-\left(b_{1}\right)_{Q}\right||f(y)| d y \\
\leq & C \sum_{k=0}^{\infty}\left(2^{-k}+2^{-k \gamma}\right) \frac{1}{\left|2^{k+1} Q\right|^{1-\delta / n}}\left(\int_{2^{k+1} Q}|f(y)|^{p} d y\right)^{1 / p} \\
& \times\left[\left|2^{k+1} Q\right|^{\beta / n+1 / p^{\prime}} \frac{1}{\left|2^{k+1} Q\right|^{\beta / n}}\left(\frac{1}{\left|2^{k+1} Q\right|} \int_{2^{k+1} Q}\left|b_{1}(y)-\left(b_{1}\right)_{2^{k+1}}\right|^{p^{\prime}} d y\right)^{1 / p^{\prime}}\right. \\
& \left.+\left|\left(b_{1}\right)_{2^{k+1}} Q^{-\left(b_{1}\right)} Q \| 2^{k+1} Q\right|^{1 / p^{\prime}}\right] \\
\leq & C \sum_{k=0}^{\infty} k\left(2^{k(-1+\delta+\beta-n / p)}+2^{k(-\gamma+\delta+\beta-n / p)}\right)|Q|^{\beta / n+1 / p^{\prime}-1+\delta / n}|| b_{1}\left\|_{\dot{\lambda}_{\beta}}\right\| f \|_{L^{p}} \\
\leq & C|Q|^{(\delta+\beta) / n-1 / p}|| b_{1}||_{\dot{\lambda}_{\beta}}|| f \|_{L^{p}} .
\end{aligned}
$$

Thus

$$
\begin{aligned}
& \frac{1}{|Q|^{1+(\delta+\beta) / n-1 / p}} \int_{Q}|C(x)| d x \\
& \leq C|| b_{1}\left\|\left.\right|_{\lambda_{\beta}}\right\| f\left\|_{L^{p}} \frac{1}{|Q|^{1+(\delta+\beta) / n-1 / p}} \int_{Q}|Q|^{(\delta+\beta) / n-1 / p} d x \leq C\right\| b_{1}\left\|{\dot{\lambda_{\beta}}}_{\beta}\right\| f \|_{L^{p}} .
\end{aligned}
$$

This completes the proof of case $m=1$.

Now, we consider the case $m \geq 2$. We write, for $\vec{b}=\left(b_{1}, \ldots, b_{m}\right)$,

$$
\begin{aligned}
& F_{t, \delta}^{\vec{b}}(f)(x)=\int_{|x-y| \leq t}\left[\prod_{j=1}^{m}\left(\left(b_{j}(x)-\left(b_{j}\right)_{Q}\right)-\left(b_{j}(y)-\left(b_{j}\right)_{Q}\right)\right)\right] f(y) \frac{\Omega(x-y)}{|x-y|^{n-1-\delta}} d y \\
& =\sum_{j=0}^{m} \sum_{\sigma \in C_{j}^{m}}(-1)^{m-j}\left(b(x)-(b)_{Q}\right)_{\sigma} \int_{|x-y| \leq t}\left(b(y)-(b)_{Q}\right)_{\sigma^{c}} f(y) \frac{\Omega(x-y)}{|x-y|^{n-1-\delta}} d y \\
& =\prod_{j=1}^{m}\left(b_{j}(x)-\left(b_{j}\right)_{Q} F_{t, \delta}(f)(x)+(-1)^{m} F_{t, \delta}\left(\prod_{j=1}^{m}\left(b_{j}(y)-\left(b_{j}\right)_{Q}\right) f\right)(x)\right. \\
& \quad+\sum_{j=1}^{m-1} \sum_{\sigma \in C_{j}^{m}}(-1)^{m-j}\left(b(x)-(b)_{Q}\right)_{\sigma} F_{t, \delta}^{\vec{b}_{\sigma^{c}}}(f)(x),
\end{aligned}
$$

thus, set $C_{0}=\mu_{\Omega, \delta}\left(\prod_{j=1}^{m}\left(b_{j}-\left(b_{j}\right)_{Q}\right) f_{2}\right)\left(x_{0}\right)$,

$$
\left|\mu_{\Omega, \delta}^{\vec{b}}(f)(x)-\mu_{\Omega, \delta}\left(\prod_{j=1}^{m}\left(b_{j}-\left(b_{j}\right)_{Q}\right) f_{2}\right)\left(x_{0}\right)\right|
$$




$$
\begin{aligned}
\leq & \left\|\prod_{j=1}^{m}\left(b_{j}(x)-\left(b_{j}\right)_{Q}\right) F_{t, \delta}(f)(x)\right\|+\sum_{j=1}^{m-1} \sum_{\sigma \in C_{j}^{m}}\left\|\left(b(x)-(b)_{Q}\right)_{\sigma} F_{t, \delta}^{\vec{b}_{\sigma^{c}}}(f)(x)\right\| \\
& +\left\|F_{t, \delta}\left(\prod_{j=1}^{m}\left(b_{j}-\left(b_{j}\right)_{Q}\right) f_{1}\right)(x)\right\|+\| F_{t, \delta}\left(\prod_{j=1}^{m}\left(b_{j}-\left(b_{j}\right)_{Q}\right) f_{2}\right)(x) \\
& -F_{t, \delta}\left(\prod_{j=1}^{m}\left(b_{j}-\left(b_{j}\right)_{Q}\right) f_{2}\right)\left(x_{0}\right) \| \\
= & S_{1}(x)+S_{2}(x)+S_{3}(x)+S_{4}(x) .
\end{aligned}
$$

For $S_{1}(x)$, for $1<p<q<n / \delta, 1 / q=1 / p-\delta / n$, by the boundness of $\mu_{\Omega, \delta}$ from $L^{p}\left(R^{n}\right)$ to $L^{q}\left(R^{n}\right)$ and Hölder's inequality with exponent $1 / q_{1}^{\prime}+\cdots+1 / q_{m}^{\prime}+1 / q=$ 1 and Lemma 1, we have

$$
\begin{aligned}
& \frac{1}{|Q|^{1+(\delta+m \beta) / n-1 / p}} \int_{Q}\left|S_{1}(x)\right| d x \\
& \leq C \frac{1}{|Q|^{1+(\delta+m \beta) / n-1 / p}} \prod_{j=1}^{m}\left(\int_{Q}\left|b_{j}(x)-\left(b_{j}\right) Q_{Q}\right|^{q_{j}^{\prime}} d x\right)^{1 / q_{j}^{\prime}}\left(\int_{Q}\left|\mu_{\Omega, \delta}\left(f_{1}\right)\right|^{q} d x\right)^{1 / q} \\
& \leq C \frac{|Q|^{m \beta / n+1 / q_{1}^{\prime}+\cdots q_{m}^{\prime}}}{|Q|^{1+(\delta+m \beta) / n-1 / p}} \prod_{j=1}^{m} \frac{1}{|Q|^{m \beta / n}}\left(\frac{1}{|Q|} \int_{Q}\left|b_{1}(x)-\left(b_{1}\right)_{Q}\right|^{q_{j}^{\prime}} d x\right)^{1 / q_{j}^{\prime}} \\
& \quad \times\left(\int_{Q}|f(x)|^{p} d x\right)^{1 / p} \\
& \leq C|| \vec{b}\left\|_{\dot{\lambda}_{\beta}}|| f\right\|_{L^{p}} .
\end{aligned}
$$

For $S_{2}(x)$, denoting $p=r t, 1<r<s<n / \delta, 1 / s=1 / r-\delta / n$, by the boundness of $\mu_{\Omega, \delta}$ from $L^{r}\left(R^{n}\right)$ to $L^{s}\left(R^{n}\right)$ and Lemma 1, we have

$$
\begin{aligned}
& \frac{1}{|Q|^{1+(\delta+m \beta) / n-1 / p}} \int_{Q}\left|S_{2}(x)\right| d x \\
& \leq C \sum_{j=1}^{m-1} \sum_{\sigma \in C_{j}^{m}} \frac{1}{|Q|^{1+(\delta+m \beta) / n-1 / p}}\left(\int_{Q}\left|b(x)-\left(b_{Q}\right)_{\sigma}\right|^{s^{\prime}} d x\right)^{1 / s^{\prime}} \\
& \quad \times\left(\left.\int_{Q} \mu_{\Omega, \delta}\left(\left(b-b_{Q}\right)_{\sigma^{c}} f\right)(x)\right|^{s} d x\right)^{1 / s} \\
& \leq C \sum_{j=1}^{m-1} \sum_{\sigma \in C_{j}^{m}} \frac{|Q|^{|\sigma| \beta / n+1 / s^{\prime}}}{|Q|^{1+(\delta+m \beta) / n-1 / p}} \frac{1}{|Q|^{|\sigma| \beta / n}}\left(\frac{1}{|Q|} \int_{Q}\left|b(x)-\left(b_{Q}\right)_{\sigma}\right|^{s^{\prime}} d x\right)^{1 / s^{\prime}}
\end{aligned}
$$




$$
\begin{aligned}
& \times\left(\int_{Q}\left|\left(b(x)-b_{Q}\right)_{\sigma^{c}} f(x)\right|^{r} d x\right)^{1 / r} \\
\leq & C \sum_{j=1}^{m-1} \sum_{\sigma \in C_{j}^{m}} \frac{|Q|^{m \beta / n+1 / s^{\prime}+1 / r t^{\prime}}}{|Q|^{1+(\delta+m \beta) / n-1 / p}}\left\|b_{\sigma}\right\|_{\dot{\lambda}_{\beta}}\left\|b_{\sigma^{c}}\right\|_{\dot{\lambda}_{\beta}}\|f\|_{L^{p}} \\
\leq & C\|\vec{b}\|_{\dot{\lambda}_{\beta}}\|f\|_{L^{p}} .
\end{aligned}
$$

For $S_{3}(x)$, for $1<r<s<n / \delta, 1 / s=1 / r-\delta / n$, by the boundness of $\mu_{\Omega, \delta}$ from $L^{r}\left(R^{n}\right)$ to $L^{s}\left(R^{n}\right)$, taking $p=r t, 1<r<p<\infty$, we have

$$
\begin{aligned}
& \frac{1}{|Q|^{1+(\delta+m \beta) / n-1 / p}} \int_{Q}\left|S_{3}(x)\right| d x \\
& \leq \frac{1}{|Q|^{(\delta+m \beta) / n-1 / p}}\left(\frac{1}{|Q|} \int_{R^{n}}\left|\mu_{\Omega, \delta}\left(\prod_{j=1}^{m}\left(\left(b_{j}-\left(b_{j}\right)_{Q}\right)\right) f \chi_{B}\right)(x)\right|^{s} d x\right)^{1 / s} \\
& \leq C \frac{1}{|Q|^{(\delta+m \beta) / n-1 / p+1 / s}}\left(\int_{Q}\left|\prod_{j=1}^{m}\left(b_{j}(x)-\left(b_{j}\right)_{Q}\right) f(x)\right|^{r} d x\right)^{1 / r} \\
& \leq C \frac{1}{|Q|^{(\delta+m \beta) / n-1 / p+1 / s}} \prod_{j=1}^{m}\left(\int_{Q}\left|b_{j}(x)-\left(b_{j}\right)_{Q}\right|^{r t_{j}} d x\right)^{1 / r t_{j}}\left(\int_{Q}|f(x)|^{r t} d x\right)^{1 / r t} \\
& \leq C|| \vec{b}\left\|_{\lambda_{\beta}}|| f\right\|_{L^{p}} .
\end{aligned}
$$

For $S_{4}(x)$, similar to the proof of $C(x)$ in the case $m=1$, we get

$$
\begin{aligned}
S_{4}(x) \leq & \left(\int_{0}^{\infty}\left[\int_{|x-y| \leq t,\left|x_{0}-y\right|>t} \frac{|\Omega(x-y)|\left|f_{2}(y)\right|}{|x-y|^{n-1-\delta}}\left|\prod_{j=1}^{m}\left(b_{j}(y)-\left(b_{j}\right)_{Q}\right)\right| d y\right]^{2} \frac{d t}{t^{3}}\right)^{1 / 2} \\
& +\left(\int_{0}^{\infty}\left[\int_{|x-y|>t,\left|x_{0}-y\right| \leq t} \frac{\left|\Omega\left(x_{0}-y\right)\right|\left|f_{2}(y)\right|}{\left|x_{0}-y\right|^{n-1-\delta}}\left|\prod_{j=1}^{m}\left(b_{j}(y)-\left(b_{j}\right)_{Q}\right)\right| d y\right]^{2} \frac{d t}{t^{3}}\right)^{1 / 2} \\
& +\left(\int _ { 0 } ^ { \infty } \left[\int_{|x-y| \leq t,\left|x_{0}-y\right| \leq t} \mid \frac{|\Omega(x-y)|}{|x-y|^{n-1-\delta}}-\frac{\left|\Omega\left(x_{0}-y\right)\right|}{\left|x_{0}-y\right|^{n-1-\delta} \mid}\right.\right. \\
& \left.\left.\times\left|\prod_{j=1}^{m}\left(b_{j}(y)-\left(b_{j}\right)_{Q}\right)\right|\left|f_{2}(y)\right| d y\right]^{2} \frac{d t}{t^{3}}\right)^{1 / 2} \\
\equiv & V_{1}+V_{2}+V_{3},
\end{aligned}
$$

Thus, we choose $1<p_{j}<\infty, j=1, \cdots, m, 1 / p_{1}+\cdots+1 / p_{m}+1 / p=1$ and get

$$
V_{1} \leq C \sum_{k=0}^{\infty} \int_{2^{k+1} Q \backslash 2^{k} Q}\left|\prod_{j=1}^{m}\left(b_{j}(y)-\left(b_{j}\right) Q\right)\right|\left|x_{0}-y\right|^{\delta} \frac{|Q|^{1 / 2 n}|f(y)|}{\left|x_{0}-y\right|^{n+1 / 2}} d y
$$




$$
\begin{aligned}
& \leq C \sum_{k=0}^{\infty} 2^{-k / 2} \frac{1}{\left|2^{k+1} Q\right|^{1-\delta / n}} \prod_{j=1}^{m}\left[\left|2^{k+1} Q\right|^{m \beta / n+1 / p^{\prime}}\left\|b_{j}\right\|_{\dot{\lambda}_{\beta}}\right. \\
&\left.+k\left|2^{k+1} Q\right|^{m \beta / n+1 / p^{\prime}}\left\|b_{j}\right\|{\dot{\dot{\lambda}_{\beta}}}\right]\|f\|_{L^{p}} \\
& \leq C \sum_{k=0}^{\infty} k 2^{k(-1 / 2+\delta+m \beta-n / p)}|Q|^{m \beta / n+1 / p^{\prime}-1+\delta / n}\|\vec{b}\|_{\dot{\lambda}_{\beta}}\|f\|_{L^{p}} \\
& \leq C|Q|^{(\delta+m \beta) / n-1 / p}\|\mid \vec{b}\|_{\dot{\lambda}_{\beta}}\|f\|_{L^{p}} .
\end{aligned}
$$

Similarly, we have $V_{2} \leq C|Q|^{(\delta+m \beta) / n-1 / p}\|\vec{b}\|_{\dot{\lambda}_{\beta}}\|f\|_{L^{p}}$. For $V_{3}$, we obtain

$$
\begin{aligned}
& V_{3} \leq C \sum_{k=0}^{\infty} \int_{2^{k+1} Q \backslash 2^{k} Q}\left|\prod_{j=1}^{m}\left(b_{j}(y)-\left(b_{j}\right) Q\right)\right|\left(\frac{|Q|^{1 / n}}{\left|x_{0}-y\right|^{n+1-\delta}}+\frac{|Q|^{\gamma / n}}{\left|x_{0}-y\right|^{n+\gamma-\delta}}\right)|f(y)| d y \\
& \leq C \sum_{k=0}^{\infty}\left(2^{-k}+2^{-k \gamma}\right) \frac{1}{\left|2^{k+1} Q\right|^{1-\delta / n}} \int_{2^{k+1} Q}\left|\prod_{j=1}^{m}\left(b_{j}(y)-\left(b_{j}\right) Q\right)\right||f(y)| d y \\
& \leq C \sum_{k=0}^{\infty} k\left(2^{k(-1+\delta+m \beta-n / p)}+2^{k(-\gamma+\delta+m \beta-n / p)}\right) \\
& \left|2^{k+1} Q\right|^{m \beta / n+1 / p^{\prime}-1+\delta / n}|| \vec{b}\left\|_{\dot{\lambda}_{\beta}}\right\| f \|_{L^{p}} \\
& \leq C|Q|^{(\delta+m \beta) / n-1 / p}\|\vec{b}\|_{\dot{\lambda}_{\beta}}|| f \|_{L^{p}} .
\end{aligned}
$$

Thus

$$
\begin{aligned}
& \frac{1}{|Q|^{1+(\delta+m \beta) / n-1 / p}} \int_{Q}\left|S_{4}(x)\right| d x \\
& \leq C\|\vec{b}\|_{\dot{\lambda}_{\beta}}\|f\|_{L^{p}} \frac{1}{|Q|^{1+(\delta+m \beta) / n-1 / p}} \int_{Q}|Q|^{(\delta+m \beta) / n-1 / p} d x \leq C\|\vec{b}\|\left\|_{\dot{\lambda}_{\beta}}\right\| f \|_{L^{p}} .
\end{aligned}
$$

This completes the proof of Theorem 1.

Proof of Theorem 2. Fix a ball $B=B(0, l)$, there exists $\epsilon_{0} \in \mathbf{Z}$ such that $2^{\epsilon_{0}-1} \leq$ $l<2^{\epsilon_{0}}$. We choose $x_{0}$ such that $2 l<\left|x_{0}\right|<3 l$. It is only to prove that

$$
2^{\epsilon_{0}\left(\alpha+n / q_{2}\right)}\left(\frac{1}{2^{\epsilon_{0} n}} \int_{|x|<2^{\epsilon_{0}}}\left|\mu_{\Omega, \delta}^{\vec{b}}(f)(x)-\mu_{\Omega, \delta}^{\vec{b}}\left(f_{2}\right)\left(x_{0}\right)\right|^{q_{2}} d x\right)^{1 / q_{2}} \leq C\|f\|_{\dot{K}_{q_{1}}^{\alpha, \infty}} .
$$

We write, for $f_{1}=f \chi_{4 B_{\epsilon_{0}}}$ and $f_{2}=f \chi_{R^{n} \backslash 4 B_{\epsilon_{0}}}$, then

$$
\left|\mu_{\Omega, \delta}^{\vec{b}}(f)(x)-\mu_{\Omega, \delta}^{\vec{b}}\left(f_{2}\right)\left(x_{0}\right)\right| \leq\left|\mu_{\Omega, \delta}^{\vec{b}}\left(f_{1}\right)(x)\right|+\left|\mu_{\Omega, \delta}^{\vec{b}}\left(f_{2}\right)(x)-\mu_{\Omega, \delta}^{\vec{b}}\left(f_{2}\right)\left(x_{0}\right)\right| .
$$


So

$$
\begin{aligned}
& 2^{\epsilon_{0}\left(\alpha+n / q_{2}\right)}\left(\frac{1}{2^{\epsilon_{0} n}} \int_{|x|<2^{\epsilon_{0}}}\left|\mu_{\Omega, \delta}^{\vec{b}}(f)(x)-\mu_{\Omega, \delta}^{\vec{b}}\left(f_{2}\right)\left(x_{0}\right)\right|^{q_{2}} d x\right)^{1 / q_{2}} \\
& \leq 2^{\epsilon_{0}\left(\alpha+n / q_{2}\right)}\left(\frac{1}{2^{\epsilon_{0} n}} \int_{|x|<2^{\epsilon_{0}}}\left|\mu_{\Omega, \delta}^{\vec{b}}\left(f_{1}\right)(x)\right|^{q_{2}} d x\right)^{1 / q_{2}} \\
& \quad+2^{\epsilon_{0}\left(\alpha+n / q_{2}\right)}\left(\frac{1}{2^{\epsilon_{0} n}} \int_{|x|<2^{\epsilon_{0}}}\left|\mu_{\Omega, \delta}^{\vec{b}}\left(f_{2}\right)(x)-\mu_{\Omega, \delta}^{\vec{b}}\left(f_{2}\right)\left(x_{0}\right)\right|^{q_{2}} d x\right)^{1 / q_{2}} \\
& =J_{1}+J_{2} .
\end{aligned}
$$

For $J_{1}$, by the ( $\left.L^{q_{1}}, L^{q_{2}}\right)$-boundedness of $\mu_{\Omega, \delta}^{\vec{b}}$ (see Lemma 4) and Lemma 2, we get

$$
\begin{aligned}
J_{1} & \leq C 2^{\epsilon_{0}\left(\alpha+n / q_{2}\right)} 2^{-\epsilon_{0} n / q_{2}}\left(\int_{R^{n}}\left|f_{1}(x)\right|^{q_{1}} d x\right)^{1 / q_{1}} \\
& \leq C 2^{\epsilon_{0} \alpha}\left\|f \chi_{B_{\epsilon_{0}}}\right\|_{L^{q_{1}}} \leq C\|f\|_{\dot{K}_{q_{1}}^{\alpha, \infty}} .
\end{aligned}
$$

For $J_{2}$, similar to the estimates of Theorem 1, we obtain using Hölder's inequality and recalling that $\max \left(-n / q_{2}-1 / 2,-n / q_{2}-\gamma\right)<\alpha \leq-n / q_{2}, 1 / q_{2}=1 / q_{1}-(\delta+$ $m \beta) / n$,

$$
\begin{aligned}
& \left|\mu_{\Omega, \delta}^{\vec{b}}\left(f_{2}\right)(x)-\mu_{\Omega, \delta}^{\vec{b}}\left(f_{2}\right)\left(x_{0}\right)\right| \\
& \leq \mid \mu_{\Omega, \delta}\left(\prod_{j=1}^{m}\left(b_{j}-\left(b_{j}\right)_{B}\right)\left(f_{2}\right)(x)-\mu_{\Omega, \delta}\left(\prod_{j=1}^{m}\left(b_{j}-\left(b_{j}\right)_{B}\right)\left(f_{2}\right)\left(x_{0}\right) \mid\right.\right. \\
& \quad+\left|\prod_{j=1}^{m}\left(b_{j}(x)-\left(b_{j}\right)_{B}\right)\right|\left|\mu_{\Omega, \delta}\left(f_{2}\right)(x)-\mu_{\Omega, \delta}\left(f_{2}\right)\left(x_{0}\right)\right|=W_{1}(x)+W_{2}(x) .
\end{aligned}
$$

For $W_{1}(x)$, similar to the proof of $S_{4}(x)$ in Theorem 1 , set $1 / v_{1}+\cdots+1 / v_{m}+$ $1 / q_{1}=1$, by the Minkowski's inequality, we have

$$
W_{1}(x) \leq V_{1}+V_{2}+V_{3} .
$$

For $V_{1}$, we have

$$
\begin{aligned}
V_{1} & \leq C \sum_{k=1}^{\infty} \int_{B_{\epsilon_{0}+k}}\left|\prod_{j=1}^{m}\left(b_{j}(y)-\left(b_{j}\right)_{Q}\right)\right| \frac{|Q|^{1 / 2 n}|f(y)|}{\left|x_{0}-y\right|^{n+1 / 2-\delta}} d y \\
& \leq C \sum_{k=1}^{\infty} \int_{B_{\epsilon_{0}+k}} \frac{2^{\epsilon_{0} / 2}}{2^{\left(\epsilon_{0}+k\right)(n+1 / 2-\delta)}}\left|\prod_{j=1}^{m}\left(b_{j}(y)-\left(b_{j}\right)_{B}\right)\right||f(y)| d y
\end{aligned}
$$




$$
\begin{aligned}
& \leq C \sum_{k=1}^{\infty} 2^{k\left(\delta+m \beta-n / q_{1}-\alpha-1 / 2\right)} 2^{\epsilon_{0}\left(\delta+m \beta-n / q_{1}-\alpha\right)}\|\vec{b}\|_{\dot{\lambda}_{\beta}} 2^{\left(\epsilon_{0}+k\right) \alpha}\left\|f \chi_{\epsilon_{0}+k}\right\|_{L^{q_{1}}} \\
& \leq C 2^{\epsilon_{0}\left(-n / q_{2}-\alpha\right)}\|\vec{b}\|_{\dot{\lambda}_{\beta}}\|f\|_{\dot{K}_{q_{1}}^{\alpha, \infty} .}
\end{aligned}
$$

Similarly, we have $V_{2} \leq C 2^{\epsilon_{0}\left(-n / q_{2}-\alpha\right)}\|\vec{b}\|_{\dot{\lambda}_{\beta}}\|f\|_{\dot{K}_{q_{1}}^{\alpha, \infty}}$.

For $V_{3}$, we obtain

$$
\begin{aligned}
V_{3} \leq & C \sum_{k=1}^{\infty} \int_{B_{\epsilon_{0}+k}}\left|\prod_{j=1}^{m}\left(b_{j}(y)-\left(b_{j}\right)_{Q}\right)\right|\left(\frac{|Q|^{1 / n}}{\left|x_{0}-y\right|^{n+1-\delta}}+\frac{|Q|^{\gamma / n}}{\left|x_{0}-y\right|^{n+\gamma-\delta}}\right)|f(y)| d y \\
\leq & C \sum_{k=1}^{\infty} \int_{B_{\epsilon_{0}+k}}\left(\frac{2^{\epsilon_{0}}}{2^{\left(\epsilon_{0}+k\right)(n+1-\delta)}}+\frac{2^{\epsilon_{0} \gamma}}{2^{\left(\epsilon_{0}+k\right)(n+\gamma-\delta)}}\right)\left|\prod_{j=1}^{m}\left(b_{j}(y)-\left(b_{j}\right)_{B}\right)\right||f(y)| d y \\
\leq & C \sum_{k=1}^{\infty}\left(2^{k\left(m \beta+n-n / q_{1}-n+\delta-1\right)}+2^{k\left(m \beta+n-n / q_{1}-n+\delta-\gamma\right)}\right) \\
& \times 2^{\epsilon_{0}\left(m \beta+n-n / q_{1}-n+\delta\right)}\|\vec{b}\|_{\dot{\lambda}_{\beta}}\left\|f \chi_{\epsilon_{0}+k}\right\|_{L^{q_{1}}} \\
\leq & C 2^{\epsilon_{0}\left(-n / q_{2}-\alpha\right)}\|\vec{b}\|_{\dot{\lambda}_{\beta}}\|f\|_{\dot{K}_{q_{1}}^{\alpha, \infty}} .
\end{aligned}
$$

For $W_{2}(x)$, we have,

$$
\begin{aligned}
& \| F_{t, \delta}\left(f_{2}\right)(x)-F_{t, \delta}\left(f_{2}\right)\left(x_{0}\right)|| \\
& \leq\left(\int_{0}^{\infty}\left[\int_{|x-y| \leq t,\left|x_{0}-y\right|>t} \frac{|\Omega(x-y)|\left|f_{2}(y)\right|}{|x-y|^{n-1-\delta}} d y\right]^{2} \frac{d t}{t^{3}}\right)^{1 / 2} \\
& \quad+\left(\int_{0}^{\infty}\left[\int_{|x-y|>t,\left|x_{0}-y\right| \leq t} \frac{\left|\Omega\left(x_{0}-y\right)\right|\left|f_{2}(y)\right|}{\left|x_{0}-y\right|^{n-1-\delta}} d y\right]^{2} \frac{d t}{t^{3}}\right)^{1 / 2} \\
& \quad+\left(\int_{0}^{\infty}\left[\int_{|x-y| \leq t,\left|x_{0}-y\right| \leq t} \frac{|\Omega(x-y)|}{|x-y|^{n-1-\delta}}-\frac{\left|\Omega\left(x_{0}-y\right)\right|}{\left|x_{0}-y\right|^{n-1-\delta}}|| f_{2}(y) \mid d y\right]^{2} \frac{d t}{t^{3}}\right)^{1 / 2} \\
& =V_{1}^{\prime}+V_{2}^{\prime}+V_{3}^{\prime} .
\end{aligned}
$$

For $V_{3}^{\prime}$, similar to the proof of $I_{3}$ in Theorem 1, we get

$$
\begin{aligned}
V_{3}^{\prime} \leq & C \sum_{k=1}^{\infty} \int_{B_{\epsilon_{0}+k}}\left(\frac{|Q|^{1 / n}}{\left|x_{0}-y\right|^{n+1-\delta}}+\frac{|Q|^{\gamma / n}}{\left|x_{0}-y\right|^{n+\gamma-\delta}}\right)|f(y)| d y \\
\leq & C \sum_{k=1}^{\infty} \int_{B_{\epsilon_{0}+k}}\left(\frac{2^{\epsilon_{0}}}{2^{\left(\epsilon_{0}+k\right)(n+1-\delta)}}+\frac{2^{\epsilon_{0} \gamma}}{2^{\left(\epsilon_{0}+k\right)(n+\gamma-\delta)}}\right) 2^{\left(\epsilon_{0}+k\right)\left(n-n / q_{1}\right)} \\
& \left(\int_{B_{\epsilon_{0}+k}}|f(y)|^{q_{1}} d y\right)^{1 / q_{1}}
\end{aligned}
$$




$$
\begin{aligned}
& \leq C \sum_{k=1}^{\infty}\left(2^{k\left(\delta-n / q_{1}-\alpha-1\right)}+2^{k\left(\delta-n / q_{1}-\alpha-\gamma\right)}\right) 2^{\epsilon_{0}\left(\delta-n / q_{1}-\alpha\right)} 2^{\left(\epsilon_{0}+k\right) \alpha}\left\|f \chi_{\epsilon_{0}+k}\right\|_{L^{q_{1}}} \\
& \leq C 2^{\epsilon_{0}\left(\delta-\alpha-n / q_{1}\right)}\|f\|_{\dot{K}_{q_{1}}^{\alpha, \infty}}
\end{aligned}
$$

The proofs of $V_{1}^{\prime}, V_{2}^{\prime}$ are similar to that of $V_{3}^{\prime}$, we omit the details. Thus, by Hölder's inequality with $1 / v_{1}+\cdots+1 / v_{m}=1$, we have

$$
\begin{aligned}
2^{\epsilon_{0}\left(\alpha+n / q_{2}\right)} & \left(\frac{1}{2^{\epsilon_{0} n}} \int_{|x|<2^{\epsilon_{0}}}\left|W_{2}(x)\right|^{q_{2}} d x\right)^{1 / q_{2}} \\
\leq & C 2^{\epsilon_{0}\left(\alpha+n / q_{2}\right)} 2^{\epsilon_{0}\left(\delta-\alpha-n / q_{1}\right)}\left(\frac{1}{2^{\epsilon_{0} n}} \int_{|x|<2^{\epsilon_{0}}}\left|\prod_{j=1}^{m}\left(b_{j}(x)-\left(b_{j}\right)_{B}\right)\right|^{q_{2}} d x\right)^{1 / q_{2}}\|f\|_{\dot{K}_{q_{1}}^{\alpha, \infty}} \\
\leq & C 2^{\epsilon_{0}\left(\alpha+n / q_{2}\right)} 2^{\epsilon_{0}\left(\delta-\alpha-n / q_{1}\right)} 2^{\epsilon_{0}\left(m \beta+n / q_{2}\left(1 / v_{1}+\cdots+1 / v_{m}\right)\right)} \\
& \times\left(\frac{1}{2^{\epsilon_{0} n}} \frac{1}{|B|^{m \beta / n}} \prod_{j=1}^{m}\left(\frac{1}{|B|} \int_{|x|<2^{\epsilon_{0}}}\left|\left(b_{j}(x)-\left(b_{j}\right)_{B}\right)\right|^{q_{2} v_{j}} d x\right)^{1 / q_{2} v_{j}}\right)\|f\|_{\dot{K}_{q_{1}}^{\alpha, \infty}} \\
\leq & C\|\vec{b}\|_{\dot{\lambda}_{\beta}}\|f\|_{\dot{K}_{q_{1}}^{\alpha, \infty} .}
\end{aligned}
$$

Thus

This completes the proof of Theorem 2.

$$
J_{2} \leq C\|\vec{b}\|_{\dot{\lambda}_{\beta}}\|f\|_{\dot{K}_{q_{1}}^{\alpha, \infty}}
$$

\section{ACKNOWLEDGEMENT}

The authors would like to express their gratitude to the referee for his comments and suggestions. The Project supported by Hunan Provincial Natural Science Foundation of China (12JJ6003) and Scientific Research Fund of Hunan Provincial Education Departments (12K017).

\section{REFERENCES}

[1] S. Chanillo, "A note on commutators," Indiana Univ. Math. J., vol. 31, no. 1, pp. 7-16, 1982.

[2] W. G. Chen, "Besov estimates for a class of multilinear singular integrals," Acta Math. Sinica, vol. 16, no. 5, pp. 613-626, 2000.

[3] G. Hu and D. C. Yang, "A variant sharp estimate for multilinear singular integral operators," Studia Math., vol. 141, no. 1, pp. 25-42, 2000.

[4] S. Janson, "Mean oscillation and commutators of singular integral operators," Ark. Math., vol. 16, no. 2, pp. 263-270, 1978.

[5] L. Z. Liu, "Triebel-lizorkin space estimates for multilinear operators of sublinear operators," Proc. Indian Acad. Sci. (Math. Sci.), vol. 113, no. 3, pp. 379-393, 2003.

[6] L. Z. Liu, "Boundedness of multilinear operator on Triebel-Lizorkin spaces," Inter. J. of Math. and Math. Sci., vol. 5, no. 2, pp. 259-272, 2004.

[7] L. Z. Liu, "The continuity of commutators on Triebel-Lizorkin spaces," Integral Equations and Operator Theory, vol. 49, no. 1, pp. 65-76, 2004.

[8] L. Z. Liu and B. S. Wu, "Weighted boundedness for commutator of Marcinkiewicz integral on some Hardy spaces," Southeast Asian Bull. of Math., vol. 28, no. 5, pp. 643-650, 2005. 
[9] Q. Lu, S. Z. Wu and D. C. Yang, "Boundedness of commutators on Hardy type spaces," Sci. in China(ser. A), vol. 45, no. 2, pp. 232-244, 2002.

[10] S. Z. Lu and D. C. Yang, "The weighted Herz type Hardy spaces and its applications," Sci. in China(ser. A), vol. 38, no. 5, pp. 662-673, 1995.

[11] Y. Lu, S. Z. Meng and Q. Wu, "Lipschitz estimates for multilinear singular integrals," Acta Math. Scientia, vol. 24(B), no. 2, pp. 291-300, 2004.

[12] M. Paluszynski, "Characterization of the Besov spaces via the commutator operator of Coifman, Rochberg and Weiss," Indiana Univ. Math. J., vol. 44, no. 1, pp. 1-17, 1995.

[13] C. Pérez and R. Trujillo-Gonzalez, "Sharp weighted estimates for multilinear commutators," $J$. London Math. Soc., vol. 65, no. 5, pp. 672-692, 2002.

[14] E. M. Stein, Harmonic analysis: real variable methods, orthogonality and oscillatory integrals, 1st ed., ser. 43. Princeton: Princeton Univ. Press, 1993, vol. 43.

[15] A. Torchinsky, Real variable methods in harmonic analysis, ser. Pure and Applied Math. New York: Academic Press, 1986, vol. 123.

[16] A. Torchinsky and S. Wang, "A note on the Marcinkiewicz integral," Colloq. Math., vol. 60/61, no. 2, pp. 235-243, 1990.

[17] D. C. Yang, "The central Campanato spaces and its applications," Approx. Theory and Appl., vol. 10, no. 1, pp. 85-99, 1994.

\section{Authors' addresses}

\section{Gu Guangze}

College of Mathematics and Econometrics, Hunan University, Changsha 410082, P. R. of China

E-mail address: dullqian@tom.com

\section{Cai Mingjie}

College of Mathematics and Econometrics, Hunan University, Changsha 410082, P. R. of China 